\title{
Article
}

\section{Global existence, uniqueness, and asymptotic behavior of solution for the Euler-Bernoulli viscoelastic equation}

\author{
Mohamed Mellah ${ }^{1, *}$ and Ali Hakem ${ }^{2}$ \\ 1 Faculty of Exact Sciences and Computer Science, Hassiba Benbouali University of Chlef, Chlef Algeria. \\ 2 Laboratory ACEDP, Djillali Liabes University, 22000 Sidi Bel Abbes, Algeria.; hakemali@yahoo.com \\ * Correspondence: m.mellah@univ-chlef.dz
}

Received: 21 April 2019; Accepted: 8 May 2019; Published: 11 May 2019.

Abstract: We study the global existence and uniqueness of a solution to an initial boundary value problem for the Euler-Bernoulli viscoelastic equation $u_{t t}+\Delta^{2} u-g_{1} * \Delta^{2} u+g_{2} * \Delta u+u_{t}=0$. Further, the asymptotic behavior of solution is established.

Keywords: Euler-Bernoulli viscoelastic equation, global existence, asymptotic behavior, memory.

MSC: 35G16, 74Dxx, 35B40.

\section{Introduction}

$\mathbf{T}$

his work is concerned with the global existence, uniqueness, and asymptotic behavior of solution for the Euler-Bernoulli viscoelastic equation

$$
\left\{\begin{array}{l}
u_{t t}+\Delta^{2} u-g_{1} * \Delta^{2} u+g_{2} * \Delta u+u_{t}=0, \quad x \in \Omega, t>0 \\
u(x, 0)=u_{0}(x), \quad u_{t}(x, 0)=u_{1}(x), \quad x \in \Omega \\
u=0, \quad \frac{\partial u}{\partial v}=0, x \in \partial \Omega, t>0
\end{array}\right.
$$

where $\Omega$ is a bounded domain of $\mathbb{R}^{n}$ with smooth boundary $\partial \Omega$, and $v$ is the unit outer normal on $\partial \Omega$. Here $g_{1}$ and $g_{2}$ are positive functions satisfying some conditions to be specified later, and

$$
g_{i} * \chi(t)=\int_{0}^{t} g_{i}(t-\tau) \chi(\tau) d \tau, \quad i=1,2 .
$$

The Euler-Bernoulli equation

$$
u_{t t}(x, t)+\Delta^{2} u(x, t)+h\left(u_{t}\right)=f(u), \quad(x, t) \in \mathbb{R}^{n} \times(0, \infty),
$$

describes the deflection $u(x, t)$ of a beam (when $n=1$ ) or a plate (when $n=2$ ), where $\Delta^{2} u:=\Delta(\Delta u)=$ $\sum_{j=1}^{n}\left(\sum_{i=1}^{n} u_{x_{i}} u_{x_{i}}\right)_{x_{j} x_{i}}$, and $h$ and $f$ represent the friction damping and the source respectively.

Lange and Menzala [1] considered

$$
u_{t t}(x, t)+\Delta^{2} u(x, t)+a(t) u_{t}(x, t)=0
$$

where $x \in \mathbb{R}^{n}, t \geq 0, a(t)=m\left(\|\nabla v(\cdot, t)\|_{L^{2}\left(\mathbb{R}^{n}\right.}^{2}\right)$ and the real-valued function $m:[0,+\infty) \rightarrow[1,+\infty)$ will be assumed to be of class $C^{1}$ satisfying the condition $m(s) \geq 1+s$ for all $s \geq 0$. They remarked that the imaginary part of the solutions of Schrödinger's equation

$$
i w_{t}=\Delta w+i m\left(\|\nabla(\operatorname{Im} w)\|_{L^{2}\left(\mathbb{R}^{n}\right.}^{2}\right) R e w=0,
$$


are precisely the solutions for (3). Then, using Fourier transform, the existence of global classical solutions and algebraic decay rate were proved for initial data whose regularity depends on the spacial dimension $n$. Messaoudi [2] studied the equation

$$
u_{t t}(x, t)+\Delta^{2} u(x, t)+a\left|u_{t}\right|^{m-2} u_{t}=b|u|^{p-2} u,
$$

where $a, b>0, p, m>2$. He established an existence result for (4) and showed that the solution continued to exist globally if $m \geq p$. If we take the viscoelastic materials into consideration, the model (2) becomes

$$
u_{t t}(x, t)+\Delta^{2} u(x, t)-\int_{0}^{t} g(t-s) \Delta^{2} u(x, s) d s+h\left(u_{t}\right)=f(u)
$$

where $g$ is so-called viscoelastic kernel. The term $\int_{0}^{t} g(t-s) \Delta^{2} u(x, s) d s$ describes the hereditary properties of the viscoelastic materials [3]. It expresses the fact that the stress at any instant $t$ depends on the past history of strains which the material has undergone from time 0 up to $t$. Tatar [4] obtained the property of the energy decay of the model (5) for $h=f=0$ and from this, we know that the term $\int_{0}^{t} g(t-s) \Delta^{2} u(x, s) d s$, similar to the friction damping, can cause the inhibition of the energy.

Messaoudi and Mukiawa [5] studied the fourth-order viscoelastic plate equation

$$
u_{t t}(x, t)+\Delta^{2} u(x, t)-\int_{0}^{t} g(t-s) \Delta^{2} u(x, s) d s=0,
$$

in the bounded domain $\Omega=(0, \pi) \times(-l, l) \subset \mathbb{R}^{2}$ with nontraditional boundary conditions. The authors established the well-posedness of the solution and a decay result.

Rivera et al. [6] investigated the plate model:

$$
u_{t t}+\Delta^{2} u-\sigma \Delta u_{t t}+\int_{0}^{t} g(t-s) \Delta^{2} u(s) d s=0,
$$

in the bounded domain $\Omega \subset \mathbb{R}^{2}$ with mixed boundary condition and suitable geometrical hypotheses on $\partial \Omega$. They established that the energy decays to zero with the same rate of the kernel $g$ such as exponential and polynomial decay. To do so in the second case they made assumptions on $g, g^{\prime}$ and $g^{\prime \prime}$ which means that $g \simeq(1+t)^{-p}$ for $p>2$. Then they obtained the same decay rate for the energy. However, their approach can not be applied to prove similar results for $1<p \leq 2$.

Cavalcanti et al. [7] investigated the global existence, uniqueness and stabilization of energy of

$$
u_{t t}+\Delta^{2} u-\int_{0}^{t} g(t-s) \Delta^{2} u(s) d s+a(t) u_{t}=0
$$

where

$$
a(t)=M\left(\int_{\Omega}|\nabla u(x, t)|^{2} d x\right) \text { with } M \in C^{1}([0,+\infty)) .
$$

By taking a bounded or unbounded open set $\Omega$ where $M(s)>m_{0}>0$ for all $s \geq 0$, the authors showed in [7] that the energy goes to zero exponentially, provided that $g$ goes to zero at the same form.

The aim of this work is to study the global existence of regular and weak solutions of problem (1) for the bounded domain, then for $\xi: \mathbb{R}^{+} \rightarrow \mathbb{R}^{+}$a increasing $C^{2}$ function such that

$$
\xi(0)=0, \quad \xi^{\prime}(0)>0, \quad \lim _{t \rightarrow+\infty} \xi(t)=+\infty, \quad \xi^{\prime \prime}(t)<0 \quad \forall t \geq 0 .
$$

the solution features the asymptotic behavior

$$
E(t) \leq E(0) e^{-\kappa \xi(t)}, \quad \forall t \geq 0,
$$

where $E(t)$ is defined in (38) and $\kappa$ is a positive constant independent of the initial energy $E(0)$. 


\section{Preliminaries and main results}

We begin by introducing some notation that will be used throughout this work. For functions $u(x, t)$, $v(x, t)$ defined on $\Omega$, we introduce

$$
(u, v)=\int_{\Omega} u(x) v(x) d x \text { and }\|u\|_{2}=\left(\int_{\Omega}|u(x)|^{2} d x\right)^{\frac{1}{2}} .
$$

Define

$$
X=\left\{u \in H_{0}^{2}(\Omega) ; \Delta^{2} u \in L^{2}(\Omega)\right\}
$$

Then, $X$ is a Hilbert space endowed with the natural inner product

$$
(u, v)_{X}=(u, v)_{H_{0}^{2}}+\left(\Delta^{2} u, \Delta^{2} v\right) .
$$

Now let us precise the hypotheses on $g_{1}$ and $g_{2}$.

(H1) $g_{1}: \mathbb{R}^{+} \rightarrow \mathbb{R}^{+}$is a bounded function satisfying

$$
g_{1}(t) \in C^{2}\left(\mathbb{R}^{+}\right) \cap L^{1}\left(\mathbb{R}^{+}\right), \quad g_{1}(0)>0 .
$$

(H2) There exist positive constants $\alpha_{1}, \alpha_{2}$ and $\alpha_{3}$ such that

$$
-\alpha_{1} g_{1}(t) \leq g_{1}^{\prime}(t) \leq-\alpha_{2} g_{1}(t), \quad \forall t \geq 0,
$$

(H3)

$$
0 \leq g_{1}^{\prime \prime}(t) \leq \alpha_{3} g_{1}(t), \quad \forall t \geq 0,
$$

(H4) $g_{2}: \mathbb{R}^{+} \rightarrow \mathbb{R}^{+}$is a bounded function satisfying

$$
g_{2}(t) \in C^{1}\left(\mathbb{R}^{+}\right) \cap L^{1}\left(\mathbb{R}^{+}\right), \quad g_{2}(0)>0 .
$$

(H5) There exist positive constants $\eta_{1}$ and $\eta_{2}$ such that

$$
-\eta_{1} g_{2}(t) \leq g_{2}^{\prime}(t) \leq-\eta_{2} g_{2}(t), \quad \forall t \geq 0,
$$

(H6)

$$
1-\int_{0}^{t}\left(g_{1}(s)+\lambda_{1}^{-1} g_{2}(s)\right) d s=l>0,
$$

where $\lambda_{1}>0$ is the first eigenvalue of the spectral Dirichlet problem

$$
\begin{gathered}
\Delta^{2} u=\lambda_{1} u \quad \text { in } \Omega, \quad u=\frac{\partial u}{\partial v}=0 \text { in } \partial \Omega, \\
\|\nabla u\|_{2} \leq \frac{1}{\sqrt{\lambda_{1}}}\|\Delta u\|_{2} .
\end{gathered}
$$

Lemma 1. For $\phi, \psi \in C^{1}([0,+\infty[, \mathbb{R})$ we have

$$
2 \int_{0}^{t} \int_{\Omega} \phi(t-s) \psi \psi^{\prime} d x d s=-\frac{d}{d t}\left((\phi \square \psi)(t)-\int_{0}^{t} \phi(s) d s\|\psi\|_{2}^{2}\right)+\left(\phi^{\prime} \square \psi\right)(t)-\phi(t)\|\phi\|_{2}^{2},
$$

where

$$
(\phi \square \psi)(t)=\int_{0}^{t} \phi(t-s)\|\psi(t)-\psi(s)\|_{2}^{2} d s .
$$

Theorem 2. Assume that $(H 1)-(H 6)$ hold, and that $\left\{u_{0}, u_{1}\right\}$ belong to $H_{0}^{2}(\Omega) \times L^{2}(\Omega)$. Then, Problem (1) admits a unique weak solution $u$ in the class

$$
u \in C^{0}\left([0, \infty) ; H_{0}^{2}(\Omega)\right) \cap C^{1}\left([0, \infty) ; L^{2}(\Omega)\right) .
$$


Moreover, for $\xi: \mathbb{R}^{+} \rightarrow \mathbb{R}^{+}$a increasing $C^{2}$ function satisfying (6) and, if $\left\|g_{1}\right\|_{L^{1}(0, \infty)}$ is sufficiently small, we have for $\kappa>0$

$$
E(t) \leq E(0) e^{-\kappa \xi(t)}, \quad \forall t \geq 0 .
$$

\section{Existence of Solutions}

In this section we first prove the existence and uniqueness of regular solutions to Problem (1). Then, we extend the same result to weak solutions using density arguments.

\subsection{Regular solutions}

Let $\left(w_{j}\right)$ be a Galerkin basis in $X$, and let $V_{m}$ be the subspace generated by the first $m$ vectors $w_{1}, \ldots, w_{m}$. We search for a function

$$
u_{m}(t)=\sum_{i=1}^{m} k_{i m}(t) w_{i}(x), m=1,2, \ldots
$$

satisfying the approximate Cauchy problem

$$
\begin{gathered}
\left(u_{m}^{\prime \prime}(t), v\right)+\left(\Delta u_{m}(t), \Delta v\right)-\int_{0}^{t} g_{1}(t-s)\left(\Delta u_{m}(s), \Delta v\right) d s \\
-\int_{0}^{t} g_{2}(t-s)\left(\nabla u_{m}(s), \nabla v\right) d s+\left(u_{m}^{\prime}(t), v\right)=0, \quad \forall v \in V_{m}, \\
u_{m}(0)=u_{0 m} \longrightarrow u_{0} \text { in } X \text { and } u_{m}^{\prime}(0)=u_{1 m} \longrightarrow u_{1} \text { in } H_{0}^{2}(\Omega) .
\end{gathered}
$$

By standard methods in differential equations, we can prove the existence of solutions to the problem (5) - (6) on $\left[0, t_{m}\right)$ with $0<t_{m}<T$. In order to extend the solution of $(7)-(8)$ to the whole $[0, T]$, we need the following priori estimate.

Estimate 1. Taking $v=2 u_{m}^{\prime}(t)$ in $(7)$, we have

$$
\begin{aligned}
& \frac{d}{d t}\left[\left\|u_{m}^{\prime}(t)\right\|_{2}^{2}+\left\|\Delta u_{m}(t)\right\|_{2}^{2}\right]+2\left\|u_{m}^{\prime}(t)\right\|_{2}^{2}-2 \int_{0}^{t} g_{1}(t-s) \Delta u_{m}(s) \cdot \Delta u_{m}^{\prime}(t) d x d s \\
& -2 \int_{0}^{t} g_{2}(t-s) \nabla u_{m}(s) \cdot \nabla u_{m}^{\prime}(t) d x d s=0 .
\end{aligned}
$$

Using Lemma 1, we obtain

$$
\begin{aligned}
& -2 \int_{0}^{t} g_{1}(t-s) \int_{\Omega} \Delta u_{m}(s) \cdot \Delta u_{m}^{\prime}(t) d x d s \\
& =\frac{d}{d t}\left\{\left(g_{1} \square \Delta u_{m}\right)(t)-\left(\int_{0}^{t} g_{1}(s) d s\right)\left\|\Delta u_{m}(t)\right\|_{2}^{2}\right\}-\left(g_{1}^{\prime} \square \Delta u_{m}\right)(t)+g_{1}(t)\left\|\Delta u_{m}(t)\right\|_{2}^{2},
\end{aligned}
$$

and

$$
\begin{aligned}
& -2 \int_{0}^{t} g_{2}(t-s) \int_{\Omega} \nabla u_{m}(s) \cdot \nabla u_{m}^{\prime}(t) d x d s \\
& =\frac{d}{d t}\left\{\left(g_{2} \square \nabla u_{m}\right)(t)-\left(\int_{0}^{t} g_{2}(s) d s\right)\left\|\nabla u_{m}(t)\right\|_{2}^{2}\right\}-\left(g_{2}^{\prime} \square \nabla u_{m}\right)(t)+g_{2}(t)\left\|\nabla u_{m}(t)\right\|_{2}^{2},
\end{aligned}
$$

Inserting Equations (10) and (11) into Equation (9) and integrating over $[0, t] \subset[0, T]$, we obtain

$$
\begin{aligned}
& \left\|u_{m}^{\prime}(t)\right\|_{2}^{2}+\left(1-\int_{0}^{t} g_{1}(s) d s\right)\left\|\Delta u_{m}(t)\right\|_{2}^{2}+\left(g_{1} \square \Delta u_{m}\right)(t)-\left(\int_{0}^{t} g_{2}(s) d s\right)\left\|\nabla u_{m}(t)\right\|_{2}^{2}+\left(g_{2} \square \nabla u_{m}\right)(t) \\
& +2 \int_{0}^{t}\left\|u_{m}^{\prime}(s)\right\|_{2}^{2} d s-\int_{0}^{t}\left(g_{1}^{\prime} \square \Delta u_{m}\right)(s) d s+\int_{0}^{t} \int_{\Omega} g_{1}(s)\left|\Delta u_{m}(s)\right|^{2} d x d s-\int_{0}^{t}\left(g_{2}^{\prime} \square \nabla u_{m}\right)(s) d s \\
& +\int_{0}^{t} \int_{\Omega} g_{2}(s)\left|\nabla u_{m}(s)\right|^{2} d x d s=\left\|u_{1 m}(t)\right\|_{2}^{2}+\left\|\Delta u_{0 m}\right\|_{2}^{2}
\end{aligned}
$$


By using the fact that

$$
\begin{aligned}
& \left(g_{1} \square \Delta u_{m}\right)(t)+\left(g_{2} \square \nabla u_{m}\right)(t)-\int_{0}^{t}\left(g_{1}^{\prime} \square \Delta u_{m}\right)(s) d s-\int_{0}^{t}\left(g_{2}^{\prime} \square \nabla u_{m}\right)(s) d s \\
& +\int_{0}^{t} \int_{\Omega} g_{1}(s)\left|\Delta u_{m}(s)\right|^{2} d x d s+\int_{0}^{t} \int_{\Omega} g_{2}(s)\left|\nabla u_{m}(s)\right|^{2} d x d s \geq 0,
\end{aligned}
$$

and

$$
\begin{aligned}
\left(1-\int_{0}^{t} g_{1}(s) d s\right)\left\|\Delta u_{m}(t)\right\|_{2}^{2}-\left(\int_{0}^{t} g_{2}(s) d s\right)\left\|\nabla u_{m}(t)\right\|_{2}^{2} & \geq\left(1-\int_{0}^{t}\left[g_{1}(s)+\lambda_{1}^{-1} g_{2}(s)\right] d s\right)\left\|\Delta u_{m}(t)\right\|_{2}^{2} \\
& \geq l\left\|\Delta u_{m}(t)\right\|_{2}^{2},
\end{aligned}
$$

Equation (12) yields

$$
\left\|u_{m}^{\prime}(t)\right\|_{2}^{2}+l\left\|\Delta u_{m}(t)\right\|_{2}^{2}+2 \int_{0}^{t}\left\|u_{m}^{\prime}(s)\right\|_{2}^{2} d s \leq\left\|u_{1 m}(t)\right\|_{2}^{2}+\left\|\Delta u_{0 m}\right\|_{2}^{2} .
$$

Taking the convergence of Equation (8) into consideration, we arrive at

$$
\left\|u_{m}^{\prime}(t)\right\|_{2}^{2}+l\left\|\Delta u_{m}(t)\right\|_{2}^{2}+2 \int_{0}^{t}\left\|u_{m}^{\prime}(s)\right\|_{2}^{2} d s \leq L_{1} .
$$

where $L_{1}=\left\|u_{1}\right\|_{2}^{2}+\left\|\Delta u_{0}\right\|_{2}^{2}$.

Estimate 2. Firstly, we obtain an estimate for $u_{m}^{\prime \prime}(0)$ in the $L^{2}$ norm. indeed, setting $v=u_{m}^{\prime \prime}(0)$ and $t=0$ in Equation (7), we obtain

$$
\left\|u_{m}^{\prime \prime}(0)\right\|_{2}^{2} \leq\left[\left\|\Delta^{2} u_{0 m}\right\|_{2}+\left\|u_{1 m}\right\|_{2}\right]\left\|u_{m}^{\prime \prime}(0)\right\|_{2} .
$$

From Equations (8), (14) and (15), it follows that

$$
\left\|u_{m}^{\prime \prime}(0)\right\|_{2} \leq L_{2}, \quad \forall m \in \mathbb{N},
$$

where $L_{2}$ is a positive constant independent of $m \in \mathbb{N}$. Differentiating Equation (7) with respect to $t$, and setting $v=u_{m}^{\prime \prime}(t)$, we obtain

$$
\begin{aligned}
& \frac{d}{d t}\left[\frac{1}{2}\left\|u_{m}^{\prime \prime}(t)\right\|_{2}^{2}+\frac{1}{2}\left\|\Delta u_{m}^{\prime}(t)\right\|_{2}^{2}\right]+\left\|u_{m}^{\prime \prime}(t)\right\|_{2}^{2} \\
= & -g_{1}(0) \int_{\Omega} \Delta^{2} u_{m}(t) u_{m}^{\prime \prime}(t) d x-\int_{\Omega} \int_{0}^{t} g_{1}^{\prime}(t-s) \Delta^{2} u_{m}(s) u_{m}^{\prime \prime}(t) d s d x \\
& -g_{2}(0) \int_{\Omega} \Delta u_{m}(t) u_{m}^{\prime \prime}(t) d x-\int_{\Omega} \int_{0}^{t} g_{2}^{\prime}(t-s) \Delta u_{m}(s) u_{m}^{\prime \prime}(t) d s d x \\
= & -g_{2}(0) \int_{\Omega} \Delta u_{m}(t) u_{m}^{\prime \prime}(t) d x-\int_{\Omega} \int_{0}^{t} g_{2}^{\prime}(t-s) \Delta u_{m}(s) u_{m}^{\prime \prime}(t) d s d x \\
& -g_{1}(0)\left\|\Delta u_{m}^{\prime}(t)\right\|_{2}^{2}+g_{1}(0) \frac{d}{d t} \int_{\Omega} \Delta u_{m}(t) \cdot \Delta u_{m}^{\prime}(t) d x \\
& +\frac{d}{d t}\left\{\int_{0}^{t} g_{1}^{\prime}(t-s) \int_{\Omega} \Delta u_{m}(t) \cdot \Delta u_{m}^{\prime}(t) d x d s\right\}-g_{1}^{\prime}(0) \int_{\Omega} \Delta u_{m}(t) \cdot \Delta u_{m}^{\prime}(t) d x \\
& -\int_{0}^{t} g_{1}^{\prime \prime}(t-s) \int_{\Omega} \Delta u_{m}(s) \cdot \Delta u_{m}^{\prime}(t) d x d s .
\end{aligned}
$$

By (H5), Hölder's inequality and Young's inequality give

$$
-\int_{\Omega} \int_{0}^{t} g_{2}^{\prime}(t-s) \Delta u_{m}(s) u_{m}^{\prime \prime}(t) d s d x \leq \frac{1}{2}\left\|u_{m}^{\prime \prime}(t)\right\|_{2}^{2}+\frac{\eta_{1}^{2}\left\|g_{2}\right\|_{L^{1}}}{2} \int_{0}^{t} g_{2}(t-s)\left\|\Delta u_{m}(s)\right\|_{2}^{2} d s .
$$


From Equation (14) we obtain

$$
-g_{2}(0) \int_{\Omega} \Delta u_{m}(t) u_{m}^{\prime \prime}(t) d x \leq \frac{1}{2}\left\|u_{m}^{\prime \prime}(t)\right\|_{2}^{2}+\frac{\left[g_{2}(0)\right]^{2} L_{1}}{2 l}
$$

and

$$
\begin{aligned}
-g_{1}^{\prime}(0) \int_{\Omega} \Delta u_{m}(t) \cdot \Delta u_{m}^{\prime}(t) d x & \leq \frac{\left|g_{1}^{\prime}(0)\right|}{2}\left(\left\|\Delta u_{m}(t)\right\|_{2}^{2}+\left\|\Delta u_{m}^{\prime}(t)\right\|_{2}^{2}\right) \\
& \leq \frac{\left|g_{1}^{\prime}(0)\right| L_{1}}{2 l}+\frac{\left|g_{1}^{\prime}(0)\right|}{2}\left\|\Delta u_{m}^{\prime}(t)\right\|_{2}^{2} .
\end{aligned}
$$

From (H3), we deduce

$$
\int_{\Omega} \int_{0}^{t} g_{1}^{\prime \prime}(t-s) \Delta u_{m}(s) \Delta u_{m}^{\prime}(t) d s d x \leq \frac{1}{2}\left\|\Delta u_{m}^{\prime}(t)\right\|_{2}^{2}+\frac{\alpha_{3}^{2}\left\|g_{1}\right\|_{L^{1}}}{2} \int_{0}^{t} g_{1}(t-s)\left\|\Delta u_{m}(s)\right\|_{2}^{2} d s
$$

Inserting Equations (18)-(21) in Equation (17), we get

$$
\begin{aligned}
\frac{1}{2}\left\|u_{m}^{\prime \prime}(t)\right\|_{2}^{2}+\frac{1}{2}\left\|\Delta u_{m}^{\prime}(t)\right\|_{2}^{2} \leq & \left\|u_{m}^{\prime \prime}(0)\right\|_{2}^{2}+\left\|\Delta u_{1 m}^{\prime}\right\|_{2}^{2}+C_{3}+g_{1}(0) \int_{\Omega} \Delta u_{m}(t) \cdot \Delta u_{m}^{\prime}(t) d x \\
& +\int_{0}^{t} g_{1}^{\prime}(t-s) \int_{\Omega} \Delta u_{m}(t) \cdot \Delta u_{m}^{\prime}(t) d x d s+C_{4} \int_{0}^{t}\left\|\Delta u_{m}^{\prime}(s)\right\|_{2}^{2} d s
\end{aligned}
$$

where

$$
C_{3}=\left[\frac{\eta_{1}^{2}\left\|g_{2}\right\|_{L^{1}}}{2}+\frac{\left[g_{2}(0)\right]^{2} L_{1}}{2 l}+\frac{\left|g_{1}^{\prime}(0)\right| L_{1}}{2 l}\right] T+\left[\frac{\eta_{1}^{2}\left\|g_{2}\right\|_{L^{1}(0, \infty)}\left\|g_{2}\right\|_{L^{\infty}(0, \infty)}}{2}+\frac{\alpha_{1}^{2}\left\|g_{1}\right\|_{L^{1}(0, \infty)}\left\|g_{1}\right\|_{L^{\infty}(0, \infty)}}{2}\right] \frac{L_{1} T}{l}
$$

and

$$
C_{4}=\frac{\left|g_{1}^{\prime}(0)\right|}{2}+\frac{1}{2}
$$

Using Hölder's inequality, we know that, for any $\delta>0$,

$$
\begin{aligned}
& g_{1}(0) \int_{\Omega} \Delta u_{m}(t) \cdot \Delta u_{m}^{\prime}(t) d x+\int_{0}^{t} g_{1}^{\prime}(t-s) \int_{\Omega} \Delta u_{m}(t) \cdot \Delta u_{m}^{\prime}(t) d x d s \\
& \leq 2 \delta\left\|\Delta u_{m}^{\prime}(t)\right\|_{2}^{2}+\frac{\left[g_{1}(0)\right]^{2}}{4 \delta}\left\|\Delta u_{m}(t)\right\|_{2}^{2}+\frac{\alpha_{1}^{2}}{4 \delta}\left\|g_{1}\right\|_{L^{1}(0, \infty)}\left\|g_{1}\right\|_{L^{\infty}(0, \infty)} \int_{0}^{t}\left\|\Delta u_{m}(s)\right\|_{2}^{2} d s \\
& \leq 2 \delta\left\|\Delta u_{m}^{\prime}(t)\right\|_{2}^{2}+C_{5},
\end{aligned}
$$

where

$$
C_{5}=\left[\frac{\left[g_{1}(0)\right]^{2}}{4 \delta}+\frac{\alpha_{1}^{2}}{4 \delta}\left\|g_{1}\right\|_{L^{1}(0, \infty)}\left\|g_{1}\right\|_{L^{\infty}(0, \infty)} T\right] \frac{L_{1}}{l} .
$$

Combining Equation (22) and Equation (23), we get

$$
\frac{1}{2}\left\|u_{m}^{\prime \prime}(t)\right\|_{2}^{2}+\left(\frac{1}{2}-2 \delta\right)\left\|\Delta u_{m}^{\prime}(t)\right\|_{2}^{2} \leq\left\|u_{m}^{\prime \prime}(0)\right\|_{2}^{2}+\left\|\Delta u_{1 m}^{\prime}\right\|_{2}^{2}+C_{3}+C_{5}+C_{4} \int_{0}^{t}\left\|\Delta u_{m}^{\prime}(s)\right\|_{2}^{2} d s,
$$

Fixing $\delta>0$, sufficiently small, so that $\frac{1}{2}-2 \delta>0$ in Equation (24), and taking into account Equations (8) and (16), we get from Gronwall's Lemma the second estimate,

$$
\left\|u_{m}^{\prime \prime}(t)\right\|_{2}^{2}+\left\|\Delta u_{m}^{\prime}(t)\right\|_{2}^{2} \leq L_{3}
$$

where $L_{3}$ is a positive constant independent of $m \in \mathbb{N}$ and $t \in[0, T]$.

Estimate 3. Let $m_{1} \geq m_{2}$ be two natural numbers, and consider $z_{m}=u_{m_{1}}-u_{m_{2}}$. Then, applying the same way 
as in the estimate 1 and observing that $\left\{u_{0 m}\right\}$ and $\left\{u_{1 m}\right\}$ are Cauchy sequence in $X$ and $H_{0}^{2}(\Omega)$, respectively, we deduce

$$
\left\|z_{m}^{\prime}(t)\right\|_{2}^{2}+\left\|\Delta z_{m}(t)\right\|_{2}^{2}+2 \int_{0}^{t}\left\|z_{m}^{\prime}(s)\right\|_{2}^{2} d s \rightarrow 0, \text { as } n \rightarrow+\infty,
$$

for all $t \in[0, T]$.

Therefore, from Equations (24), (25) and (26), we deduce that there exist a subsequence $\left\{u_{\mu}\right\}$ of $\left\{u_{m}\right\}$ and $u$ such that

$$
\begin{gathered}
u_{\mu}^{\prime} \rightarrow u^{\prime} \text { strongly in } C^{0}\left([0, T] ; L^{2}(\Omega)\right), \\
u_{\mu} \rightarrow u \text { strongly in } C^{0}\left([0, T] ; H_{0}^{2}(\Omega)\right), \\
u_{\mu}^{\prime \prime} \rightarrow u^{\prime \prime} \text { weakly star in } L^{\infty}\left(0, T ; L^{2}(\Omega)\right) .
\end{gathered}
$$

The above convergences (27)-(29) are enough to pass to the limit in Equation (7), to obtain

$$
\begin{aligned}
& u^{\prime \prime}+\Delta^{2} u-\int_{0}^{t} g_{1}(t-s) \Delta^{2} u(s) d s+\int_{0}^{t} g_{2}(t-s) \Delta u(s) d s+u^{\prime}=0 \text { in } L^{\infty}\left(0, \infty ; L^{2}(\Omega)\right) \\
& u(0)=u_{0}, \quad u^{\prime}(0)=u_{1} .
\end{aligned}
$$

Next, we want to show the uniqueness of solution of (7)-(8). Let $u^{(1)}, u^{(2)}$ be two solutions of (7)-(8). Then $z=u^{(1)}-u^{(2)}$ satisfies

$$
\begin{gathered}
\left(z^{\prime \prime}(t), v\right)+(\Delta z(t), \Delta v)-\int_{0}^{t} g_{1}(t-s)(\Delta z(s), \Delta v) d s-\int_{0}^{t} g_{2}(t-s)(\nabla z(s), \nabla v) d s+\left(z^{\prime}(t), v\right)=0, \quad \forall v \in H_{0}^{2}(\Omega), \\
z(x, 0)=z^{\prime}(x, 0)=0, \quad x \in \Omega, \\
z=0, \quad \frac{\partial z}{\partial v}=0, \quad x \in \partial \Omega, t>0 .
\end{gathered}
$$

Setting $v=2 z^{\prime}(t)$ in (30), then as in deriving (14), we see that

$$
\left\|z^{\prime}(t)\right\|_{2}=\|\Delta z(t)\|_{2}=0 \text { for all } t \in[0, T]
$$

Therefore, we have the uniqueness.

\subsection{Weak solutions}

Let $\left(u_{0}, u_{1}\right) \in H_{0}^{2}(\Omega) \times L^{2}(\Omega)$. Then, since $X \times H_{0}^{2}(\Omega)$ is dense in $H_{0}^{2}(\Omega) \times L^{2}(\Omega)$ there exists $\left(u_{0 \mu}, u_{1 \mu}\right) \subset$ $X \times H_{0}^{2}(\Omega)$ such that

$$
u_{0 \mu} \rightarrow u_{0} \text { in } H_{0}^{2}(\Omega) \text { and } u_{1 \mu} \rightarrow u_{1} \text { in } L^{2}(\Omega) .
$$

Then, for each $\mu \in \mathbb{N}$, there exists a unique regular solution $u_{\mu}$ of Problem (1) in the class

$$
u_{\mu} \in L^{\infty}\left(0, \infty ; H_{0}^{2}(\Omega)\right), \quad u_{\mu}^{\prime} \in L^{\infty}\left(0, \infty ; H_{0}^{2}(\Omega)\right), \quad u_{\mu}^{\prime \prime} \in L^{\infty}\left(0, \infty ; L^{2}(\Omega)\right) .
$$

In view of Equation (33) and using an analogous argument to that in Estimate 1 and Estimate 3, we find a sequence $\left\{u_{\mu}\right\}$ of solutions to Problem (1) such that

$$
\begin{aligned}
& u_{\mu}^{\prime} \rightarrow u^{\prime} \text { weak star in } L^{\infty}\left(0, T ; L^{2}(\Omega)\right), \\
& u_{\mu} \rightarrow u \text { weak star in } L^{\infty}\left(0, T ; H_{0}^{2}(\Omega)\right), \\
& u_{\mu} \rightarrow u \text { strongly in } C^{0}\left([0, T] ; H_{0}^{2}(\Omega)\right), \\
& u_{\mu}^{\prime} \rightarrow u^{\prime} \text { strongly in } C^{0}\left([0, T] ; L^{2}(\Omega)\right),
\end{aligned}
$$


The convergences (33)-(36) are sufficient to pass to the limit in order to obtain a weak solution of Problem (1), which satisfies

$$
\begin{aligned}
& u^{\prime \prime}+\Delta^{2} u-\int_{0}^{t} g_{1}(t-s) \Delta^{2} u(s) d s+\int_{0}^{t} g_{2}(t-s) \Delta u(s) d s+u^{\prime}=0 \text { in } L^{2}\left(0, \infty ; H^{-2}(\Omega)\right), \\
& u(0)=u_{0}, \quad u^{\prime}(0)=u_{1} .
\end{aligned}
$$

The uniqueness of weak solutions requires a regularization procedure and can be obtained using the standard method of Visik-Ladyzhenskaya, c.f. Lions and Magenes [8, Chap. 3, Sec. 8.2.2].

\section{Asymptotic Behaviour}

In this section, we discuss the asymptotic behavior of the above-mentioned weak solutions. Let us define the energy associated to Problem (1) as

$$
\begin{aligned}
E(t)= & \frac{1}{2}\left\|u_{t}(t)\right\|_{2}^{2}+\frac{1}{2}\left(1-\int_{0}^{t} g_{1}(s) d s\right)\|\Delta u(t)\|_{2}^{2}+\frac{1}{2}\left(g_{1} \square \Delta u\right)(t) \\
& -\frac{1}{2}\left(\int_{0}^{t} g_{2}(s) d s\right)\|\nabla u(t)\|_{2}^{2}+\frac{1}{2}\left(g_{2} \square \nabla u\right)(t) .
\end{aligned}
$$

To demonstrate our decay result, the lemmas below are essential.

Lemma 3. For any $t>0$

$$
0 \leq E(t) \leq \frac{1}{2}\left[\left\|u_{t}(t)\right\|_{2}^{2}+\|\Delta u(t)\|_{2}^{2}+\left(g_{1} \square \Delta u\right)(t)+\left(g_{2} \square \nabla u\right)(t)\right] .
$$

Proof. Using the fact that $\|\nabla u(t)\|_{2}^{2} \leq \lambda_{1}^{-1}\|\Delta u(t)\|_{2}^{2}$, we have

$$
\begin{aligned}
& \left(1-\int_{0}^{t} g_{1}(\tau) d \tau\right)\|\Delta u(t)\|_{2}^{2}-\left(\int_{0}^{t} g_{2}(\tau) d \tau\right)\|\nabla u(t)\|_{2}^{2} \\
& \geq\left(1-\int_{0}^{t}\left[g_{1}(s)+\lambda_{1}^{-1} g_{2}(s)\right] d s\right)\|\Delta u(t)\|_{2}^{2}
\end{aligned}
$$

and according to (H6) we have $E(t) \geq 0$,

and

$$
\begin{aligned}
E(t)= & \frac{1}{2}\left\|u_{t}(t)\right\|_{2}^{2}+\frac{1}{2}\|\Delta u(t)\|_{2}^{2}+\frac{1}{2}\left(g_{1} \square \Delta u\right)(t)+\frac{1}{2}\left(g_{2} \square \nabla u\right)(t) \\
& -\frac{1}{2}\left\{\left(\int_{0}^{t} g_{1}(s) d s\right)\|\Delta u(t)\|_{2}^{2}+\left(\int_{0}^{t} g_{2}(s) d s\right)\|\nabla u(t)\|_{2}^{2}\right\} \\
\leq & \frac{1}{2}\left[\left\|u_{t}(t)\right\|_{2}^{2}+\|\Delta u(t)\|_{2}^{2}+\left(g_{1} \square \Delta u\right)(t)+\left(g_{2} \square \nabla u\right)(t)\right] .
\end{aligned}
$$

Lemma 4. The energy $E(t)$ satisfies

$$
E^{\prime}(t) \leq-\left\|u_{t}(t)\right\|_{2}^{2}-\frac{1}{2} \alpha_{2}\left(g_{1} \square \Delta u\right)(t)-\frac{1}{2} \eta_{2}\left(g_{2} \square \nabla u\right)(t)-\frac{1}{2}\left[g_{1}(0)-\alpha_{1}\left\|g_{1}\right\|_{L^{1}(0, \infty)}\right]\|\Delta u(t)\|_{2}^{2} \leq 0 .
$$

Proof. Multiplying the first equation in (1) by $u_{t}$ and integrating over $\Omega$, we obtain $\frac{d}{d t}\left[\frac{1}{2}\left\|u_{t}(t)\right\|_{2}^{2}+\frac{1}{2}\|\Delta u(t)\|_{2}^{2}\right]+\left\|u_{t}(t)\right\|_{2}^{2}=\int_{0}^{t} g_{1}(t-\tau) \Delta u(\tau) \cdot \Delta u_{t}(t) d x d \tau+\int_{0}^{t} g_{2}(t-\tau) \nabla u(\tau) \cdot \nabla u_{t}(t) d x d \tau$. 
Exploiting (10)-(11) and by (H1) - (H5), we deduce

$$
\begin{aligned}
E^{\prime}(t) & =-\left\|u_{t}(t)\right\|_{2}^{2}+\frac{1}{2}\left(g_{1}^{\prime} \square \Delta u\right)(t)-\frac{1}{2} g_{1}(t)\|\Delta u(t)\|_{2}^{2}+\frac{1}{2}\left(g_{2}^{\prime} \square \nabla u\right)(t)-\frac{1}{2} g_{2}(t)\|\nabla u(t)\|_{2}^{2} \\
& \leq-\left\|u_{t}(t)\right\|_{2}^{2}-\frac{1}{2} \alpha_{2}\left(g_{1} \square \Delta u\right)(t)-\frac{1}{2} \eta_{2}\left(g_{2} \square \nabla u\right)(t)-\frac{1}{2} g_{1}(t)\|\Delta u(t)\|_{2}^{2} .
\end{aligned}
$$

From assumptions $(H 2)$ and since $\int_{0}^{t} g_{1}^{\prime}(\tau) d \tau=g_{1}(t)-g_{1}(0)$, we obtain

$$
\begin{aligned}
-\frac{1}{2} g_{1}(t)\|\Delta u(t)\|_{2}^{2} & =-\frac{1}{2} g_{1}(0)\|\Delta u(t)\|_{2}^{2}-\frac{1}{2}\left(\int_{0}^{t} g_{1}^{\prime}(s) d s\right)\|\Delta u(t)\|_{2}^{2} \\
& \leq-\frac{1}{2} g_{1}(0)\|\Delta u(t)\|_{2}^{2}+\frac{\alpha_{1}}{2}\left\|g_{1}\right\|_{L^{1}(0, \infty)}\|\Delta u(t)\|_{2}^{2} \\
& =-\frac{1}{2}\left[g_{1}(0)-\alpha_{1}\left\|g_{1}\right\|_{L^{1}(0, \infty)}\right]\|\Delta u(t)\|_{2}^{2} .
\end{aligned}
$$

Combining Equation (40) and Equation (41), we conclude that

$$
\begin{aligned}
E^{\prime}(t) \leq & -\left\|u_{t}(t)\right\|_{2}^{2}-\frac{1}{2} \alpha_{2}\left(g_{1} \square \Delta u\right)(t)-\frac{1}{2} \eta_{2}\left(g_{2} \square \nabla u\right)(t) \\
& -\frac{1}{2}\left[g_{1}(0)-\alpha_{1}\left\|g_{1}\right\|_{L^{1}(0, \infty)}\right]\|\Delta u(t)\|_{2}^{2} \leq 0 .
\end{aligned}
$$

Multiplying Equation (39) by $e^{\kappa((t)}(\kappa>0)$ and utilizing Lemma 3, we have

$$
\begin{aligned}
\frac{d}{d t}\left(e^{\kappa \xi(t)} E(t)\right) \leq & -e^{\kappa \xi(t)} E(t)\left\|u_{t}(t)\right\|_{2}^{2}-\frac{1}{2} \alpha_{2}\left(g_{1} \square \Delta u\right)(t) e^{\kappa \xi(t)} E(t)-\frac{1}{2} \eta_{2}\left(g_{2} \square \nabla u\right)(t) e^{\kappa \xi(t)} E(t) \\
& -\frac{1}{2}\left[g_{1}(0)-\alpha_{1}\left\|g_{1}\right\|_{L^{1}(0, \infty)}\right] e^{\kappa \xi(t)} E(t)\|\Delta u(t)\|_{2}^{2}+\kappa \xi^{\prime}(t) e^{\kappa \xi(t)} E(t) \\
\leq & -\frac{1}{2}\left[2-\kappa \xi^{\prime}(t)\right] e^{\kappa \xi(t)} E(t)\left\|u_{t}(t)\right\|_{2}^{2}-\frac{1}{2}\left[\alpha_{2}-\kappa \xi^{\prime}(t)\right] e^{\kappa \xi(t)} E(t)\left(g_{1} \square \Delta u\right)(t) \\
& -\frac{1}{2}\left[\eta_{2}-\kappa \xi^{\prime}(t)\right] e^{\kappa \xi(t)} E(t)\left(g_{2} \square \nabla u\right)(t) \\
& -\frac{1}{2}\left[g_{1}(0)-\alpha_{1}\left\|g_{1}\right\|_{L^{1}(0, \infty)}-\kappa \xi^{\prime}(t)\right] e^{\kappa \xi(t)} E(t)\|\Delta u(t)\|_{2}^{2} .
\end{aligned}
$$

Using the fact that $\xi^{\prime}$ is decreasing we arrive at

$$
\begin{aligned}
\frac{d}{d t}\left(e^{\kappa \xi(t)} E(t)\right) \leq & -\frac{1}{2}\left[2-\kappa \xi^{\prime}(0)\right] e^{\kappa \xi(t)} E(t)\left\|u_{t}(t)\right\|_{2}^{2}-\frac{1}{2}\left[\alpha_{2}-\kappa \xi^{\prime}(0)\right] e^{\kappa \xi(t)} E(t)\left(g_{1} \square \Delta u\right)(t) \\
& -\frac{1}{2}\left[\eta_{2}-\kappa \xi^{\prime}(0)\right] e^{\kappa \xi(t)} E(t)\left(g_{2} \square \nabla u\right)(t) \\
& -\frac{1}{2}\left[g_{1}(0)-\alpha_{1}\left\|g_{1}\right\|_{L^{1}(0, \infty)}-\kappa \xi^{\prime}(0)\right] e^{\kappa \xi(t)} E(t)\|\Delta u(t)\|_{2}^{2} .
\end{aligned}
$$

Choosing $\left\|g_{1}\right\|_{L^{1}(0, \infty)}$ sufficiently small so that

$$
g_{1}(0)-\alpha_{1}\left\|g_{1}\right\|_{L^{1}(0, \infty)}=L>0,
$$

and choosing $\kappa$ sufficiently small in order to have

$$
2-\kappa \xi^{\prime}(0)>0, \quad \alpha_{2}-\kappa \xi^{\prime}(0)>0, \quad \eta_{2}-\kappa \xi^{\prime}(0)>, \quad L-\kappa \xi^{\prime}(0)>0 .
$$

from Equation (43) we arrive at

$$
\frac{d}{d t}\left(e^{\kappa \xi(t)} E(t)\right) \leq 0, \quad t>0
$$

Integrating the above inequality over $(0, t)$, it follows that

$$
E(t) \leq E(0) e^{-\kappa \xi(t)}, \quad t>0 .
$$


Author Contributions: All authors contributed equally to the writing of this paper. All authors read and approved the final manuscript.

Conflicts of Interest: "The authors declare no conflict of interest."

\section{References}

[1] Lange, H., \& Menzala, G. P. (1997). Rates of decay of a nonlocal beam equation. Differential and Integral Equations, 10(6), 1075-1092.

[2] Messaoudi, S. A. (2002). Global existence and nonexistence in a system of Petrovsky. Journal of Mathematical Analysis and Applications, 265(2), 296-308.

[3] Fabrizio, M., \& Morro, A. (1992). Mathematical problems in linear viscoelasticity (Vol. 12). Siam.

[4] Tatar, N. E. (2011). Arbitrary decays in linear viscoelasticity. Journal of Mathematical Physics, 52(1), 013502.

[5] S. A. Messaoudi, Mukiawa, SE: Existence and general decay of a viscoelastic plate equation. Electron. J. Differ. Equ. 2016, 22 (2016).

[6] Rivera, J. M., Lapa, E. C., \& Barreto, R. (1996). Decay rates for viscoelastic plates with memory. Journal of elasticity, 44(1), 61-87.

[7] Cavalcanti, M. M., Cavalcanti, V. D., \& Ma, T. F. (2004). Exponential decay of the viscoelastic Euler-Bernoulli equation with a nonlocal dissipation in general domains. Differential and Integral Equations, 17(5-6), 495-510.

[8] J. L. Lions and E. Magenes, Problèemes aux Limites non Homogènes, Aplications, Vol. 1 (Dunod, Paris, 1968).

(C) 2019 by the authors; licensee PSRP, Lahore, Pakistan. This article is an open access article distributed under the terms and conditions of the Creative Commons Attribution (CC-BY) license (http://creativecommons.org/licenses/by/4.0/). 